\title{
Zarathoustra aux îles : Vasco (Chadourne, 1927) et Touriste de bananes (Simenon, 1938)
}

\author{
Florence LOJACONO \\ Universidad de Las Palmas de Gran Canaria \\ flojacono@gmail.com
}

Recibido: $26 / 05 / 2016$

Aceptado: 27/09/2016

Résumé

En cette période de l'entre-deux-guerres, Marc Chadourne et Georges Simenon ont de nombreux intérêts communs : voyages, lectures et, bien sûr, le grand reportage littéraire. Dans cet article nous irons à Tahiti, où ont séjourné les deux auteurs, grâce à Vasco de Chadourne et à Touriste de bananes de Simenon. Dans les deux romans il s'agit de suivre le parcours d'un protagoniste qui, fuyant la France, aborde les rivages d'une Polynésie qu'il pense être le cadre propice de sa renaissance. Nous verrons de quelle manière, dans ces deux textes, surgit le désir de l'île comme remède au spleen d'une part et comme antidote au nihilisme d'autre part. Même si l'exotisme est au rendez-vous, la lumière étincelante d'une nature intacte se révèle incapable de chasser les vieilles lâchetés, les plages immaculées ne peuvent endiguer les vagues d'angoisse qui les submergent de plus en plus souvent. Jusqu'à l'épuisement. Jusqu'au suicide.

Mots clés : Chadourne, Simenon, Nietzsche, Tahiti.

\section{Zarathoustra en las islas: Vasco (Chadourne, 1927) y Touriste de bananes}

(Simenon, 1938)

\section{Resumen}

En el periodo de entreguerras, Marc Chadourne y Georges Simenon compartieron varias aficiones: viajar, leer y, por supuesto, el reportaje literario. En este artículo, gracias a Vasco de Chadourne y a Touriste de Bananes de Simenon, nos desplazaremos a Tahití, donde residieron ambos autores. En las dos novelas, seguiremos a unos protagonistas que abandonan la Francia continental para asentarse en la Polinesia, a la que consideran el entorno ideal para renacer. Veremos cómo en ambos textos se anhela la isla como remedio al spleen y, a la vez, como antídoto contra el nihilismo. A pesar del exotismo del lugar y de la luz que centellea sobre una naturaleza intacta, no logran alejar de sus vidas las viejas cobardías. Las playas inmaculadas no pueden con las olas de angustia que los hunden cada vez más. Hasta el agotamiento. Hasta el suicido.

Palabras clave: Chadourne, Simenon, Nietzsche, Tahití. 


\title{
Zarathoustra to the Islands: Vasco (Chadourne, 1927) and Touriste de bananes (Simenon, 1938)
}

\begin{abstract}
In the interwar period, Marc Chadourne and Georges Simenon had many common interests: travel, readings and, of course, literary reportage. In this paper we will travel to Tahiti-where both authors lived - through the works Vasco by Chadourne, and Touriste de bananes by Simenon. Each novel follows the path of a man who, escaping from France, reaches the shores of Polynesia, a land he thinks will be the fertile ground for his rebirth. We will see how, in both novels, the desire for the island ariseson the one hand as a drug against spleen, and on the other as an antidote to nihilism. In spite of the island's exoticism, the sparkling light over its unspoilt nature cannot possibly dispel the cowardice latent in their lives. The immaculate beaches are powerless to quell their pangs of anxiety, and the protagonists are increasingly overwhelmed by angst ; until exhaustion sets in. And then, suicide.
\end{abstract}

Key words : Chadourne, Simenon, Nietzsche, Tahiti.

Sommaire : 1. Le contexte littéraire. 2. Généalogie du désir insulaire. 3. Zarathoustra aux îles. 4. Conclusion.

\section{Referencia normalizada}

Lojacono, F. (2016). «Zarathoustra aux îles : Vasco (Chadourne, 1927) et Touriste de bananes (Simenon, 1938)». Thélème. Revista Complutense de Estudios Franceses, Vol. 31, Núm. 2: 283-296. http://dx.doi.org/10.5209/rev_THEL.2016.v31.n2.52786

\section{Le contexte littéraire}

Marc Chadourne (1895-1975) et Georges Simenon (1903-1989), deux écrivains contemporains de langue française, l'un français et l'autre belge, ont connu des trajectoires similaires. Ces deux lecteurs passionnés de Joseph Conrad ont largement contribué à un genre très en vogue entre les deux guerres : le grand reportage. Le goût pour le grand reportage littéraire s'est développé en France dans une parenthèse idéalement ouverte par le roman de Conrad Au cœur des ténèbres (1902) et refermée par le Voyage au bout de la nuit de Céline (1932). C'est ainsi qu'André Gide publie Voyage au Congo en 1927 suivi de Retour du Tchad en 1928, Paul Morand ParisTombouctou en 1928 et Albert Londres Terre d'ébène en 1929. Dans les années trente la production est florissante, ce que souligne Chadourne dans un article qu'il écrit justement sur Le Retour du Tchad, quand il affirme que la relation de voyage « prend dans la production actuelle une place de plus en plus considérable » (Chadourne, 1928: 858). Les quotidiens de l'époque font alors souvent appel pour leurs publications à des écrivains reconnus comme Saint-Exupéry, Cendras ou Cocteau (Boucharenc, $2004: 30$ ). Chadourne, considéré à l'époque comme « le français ayant le plus voyagé » (Pittman, $2010: 158$ ), reçoit en 1931 le grand prix du reportage pour Chine. La même année Gallimard lance Voilà, un hebdomadaire consacré au grand 
reportage auquel collaborera Simenon avec, entre autres, en octobre et novembre 1932, une série d'articles directement inspirés de son expérience africaine et dont la formule choc « l'Afrique, ça n'existe pas » annonce la morale de ses romans tahitiens : « l'exotisme ça n'existe pas » (Simenon, 2003b : 33).

Au point de vue personnel aussi, il y a des points communs entre les deux hommes. Ils ont vécu aux États-Unis à peu près à la même époque, et ont tous deux connu la Polynésie française, à dix ans d'intervalle. Chadourne est nommé chef de Cabinet du Gouverneur des Établissements français d'Océanie puis administrateur des colonies des Îles Sous le Vent (1921-1924). Simenon, lui, n'y séjourne que peu de temps lors de son tour du monde effectué entre décembre 1934 et mai 1935. S'ils voyagent ainsi d'un bout du monde à l'autre, entre écriture et conférences, ce qu'ils partagent surtout, c'est le même regard qu'ils portent sur les colonies, mi-triste, mi-désabusé. Entre les deux guerres, le mythe des Mers du Sud, de Robinson et de son autarcie heureuse, est encore très vivace. La dimension rêvée et désirée de l'île, modelée par Bougainville et surtout par le célèbre Post-scriptum sur l'Isle de la Nouvelle Cythère ou Tayti de Commerson, publié au Mercure de France en novembre $1769^{1}$, a été véhiculée au cours des siècles et jusqu'aux années trente par les peintres (Gauguin, Matisse), les cinéastes (Murnau), les navigateurs (London, Gerbault), les amateurs de vie naturelle (Heyerdahl), et, bien sûr, par les écrivains (Loti, Stevenson, Segalen, Dorsenne). C'est en Polynésie française, dans l'ailleurs absolu (Bachimon, 2004) que se situent les deux œuvres dont nous allons cerner, sous l'apparente douceur des palmes, la dureté des implications philosophiques. Rappelons tout d'abord brièvement les trames narratives.

Vasco (ci-après VS), le premier roman de Marc Chadourne, connut un succès immense à sa publication en 1927. Vasco, en référence à Vasco de Gama, est le surnom donné à Philippe par ses amis de jeunesse, alors étudiants comme lui à Paris, car il citait sans cesse un vers du poème de Mallarmé (1966: 99) intitulé Au seul souci de voyager (cf. Pascal Durand [2000] pour l'analyse de l'emploi du nom Vasco dans la poésie de Mallarmé). Bouleversé par la Grande Guerre, Vasco se lance dans une fuite en avant désespérée, de Paris à Tahiti où il a été nommé gérant des Comptoirs Pacifique-Sud, puis de Tahiti aux Tuamotu et enfin, des Tuamotu aux Marquises, dans la croyance toujours renouvelée en une solitude rédemptrice. La distance qu'il s'efforce de mettre entre son destin et lui va de pair avec la détérioration de sa situation aussi bien financière, suite à la gestion nonchalante des Comptoirs, qu'existentielle. Toujours assoiffé d'une paix intérieure qu'aucun paysage ne semble être capable d'assouvir, il a cru pouvoir surmonter le poids de son hérédité et tromper « l'esprit saturnien qui [...] veillait en embuscade sur cette ère de loisirs et d'oubli » (VS : 154) en se liant avec un personnage équivoque et charismatique du nom de Plessis. Mais Vasco n'aura jamais la force d'un Plessis et c'est perclus de remords et

\footnotetext{
${ }^{1}$ Ce texte est conservé à la Bibliothèque centrale du Muséum national d'Histoire naturelle de Paris (Laissus, 1978 : 152).
} 
de rage envers lui-même qu'il rentrera en France avec le sentiment amer de ne jamais avoir été à la hauteur : la mesquinerie de sa vie n'a pas disparue sous les tropiques, elle s'y est au contraire déclarée comme une lèpre aveuglante.

Inspiré par son tour du monde, Simenon écrit divers reportages et six romans : Quartier nègre (1935), Long cours (1936), Ceux de la soif (1938), Touriste de bananes (1938), L'aîné des Ferchaud (1945), Le passager clandestin (1947). Dans Touriste de bananes ou les dimanches de Tahiti (ci-après TB) le lecteur retrouve la famille Donadieu, déjà rencontrée en 1937 dans Le Testament Donadieu. Touriste de bananes n'est pas un roman policier, c'est un roman dur, « un roman roman » comme les appelait l'écrivain liégeois, c'est-à-dire un roman dans lequel l'auteur se permet de conduire le protagoniste au bout de son destin. Oscar fuit sa famille bourgeoise pour mener au sein d'une nature qu'il veut croire édénique, la vie de celui qu'il aurait voulu être à La Rochelle. Mais il ne sera qu'un « touriste de bananes » parmi d'autres, et même, plus malchanceux que tous ses prédécesseurs. Quand l'échec de sa tentative lui apparaîtra dans toute son ampleur, il se suicidera. Le lecteur intéressé par les liens entre exotisme et insularité pourra se référer à un article de 2007, Rien de nouveau sous le soleil (Lojacono), qui souligne plus particulièrement les relations entre Touriste de bananes et le primitivisme insulaire.

Une première lecture pourrait ajouter Vasco et Touristes de bananes à la liste des romans coloniaux qui furent nombreux entre les deux guerres, si l'on admet avec Lecarme que pour qu'il y ait roman colonial :

[...] il faut que l'action se situe dans une colonie, en général visitée par l'écrivain, que soit mis en scène un colonisateur, lui-même mis en relation avec des colonisés, occupant le premier plan ou l'arrièreplan. Les relations de domination, les conflits des modes de vie ou des modes de culture seront au moins évoqués: les valeurs du colonialisme seront prônées ou combattues, thématisées ou problématisées (Lecarme, $1989: 179$ ).

C'est bien, en effet, le cas de Vasco et de Touriste de bananes : l'action se déroule en Polynésie française et sont évoqués les abus de pouvoir et les malversations des colons, tout comme l'exploitation des ressources humaines et naturelles de la Polynésie. Cependant, en ce qui concerne les deux protagonistes, c'est la volonté de mettre le plus de distance possible entre le «moi » qu'ils étaient en France et le «moi » qu'ils espèrent devenir à Tahiti qui les a conduits sur ces rives solitaires, non la perspective d'un enrichissement quelconque. Et s'ils sont bien en quête d'une certaine domination, c'est uniquement à la domination de leur propre faiblesse à laquelle ils aspirent. On peut donc dire comme Simenon: "Ce ne sont pas des histoires coloniales $[\ldots]$ ce sont des histoires de gens qui sont partis pleins de sève, de vie, d'espoirs, de projets et que les tropiques ont réduit à un état que... » (Simenon, 2003b : 16). La situation administrative de colonie ne fait que précipiter la brutale prise de contact avec la réalité, renforcer l'antinomie entre le rêve édénique qui avait motivé le départ et le long cortège de petites humiliations qui s'est avéré être le quotidien des protagonistes. Les colons présents dans les deux récits, par leur cynisme 
et leur persiflage, jouent le rôle d'un chœur de cassandres entourant le déroulement de la tragédie de ses chants funestes (Alavoine, 1998 : 92).

Si la critique a parfois placé Touriste de bananes parmi les romans coloniaux, d'autres comme Alain Bertrand et Maurice Piron y voient plutôt un roman de la destinée. Dans un roman de la destinée, un évènement fortuit révèle la précarité d'une situation basée sur l'observance de règles auxquelles le protagoniste est étranger. C'est ainsi qu'un premier élément perturbateur a chassé les protagonistes hors de leur famille à la poursuite d'un nouvel équilibre, dont eux-mêmes dicteraient les règles cette fois. Débarqués à Tahiti, un second élément va venir perturber leur rêve de vie recommencée : la rencontre avec les réalités locales et, plus douloureusement encore, la rencontre avec eux-mêmes. Une fois de plus la solution sera la fuite, toujours plus loin : dans les districts pour Oscar, des Tuamotu aux Marquises pour Vasco. Mais l'éloignement n'est décidément pas la clé du problème, il en est même tout le contraire. Car seuls, à l'abri des règles de l'Autre, ils n'en continuent pas moins d'observer ces règles prescrites par d'autres pour une vie dont ils ne sont finalement pas plus les maîtres à Tahiti qu'en métropole. Vasco et Oscar se sentent différents d'une famille au sein de laquelle ils ne reconnaissent aucune de leurs aspirations. Sur le terrain favorable de cette inadaptation première, la nonchalance des îles ne fera qu'accélérer le dénouement. Le destin d'Oscar et de Vasco est scellé au creux du même sillage le jour même de leur arrivée à Tahiti, car ils n'ont de goût « que pour l'inquiétude et la chimère » (VS : 135). Désirant renaître plus forts sous des cieux plus hospitaliers, ils croient conjurer leur faiblesse par un éloignement qui ne fait que la souligner. Incapables de jouir du présent, ils sont gouvernés par les démons du passé qui introduisent le soupçon dans la clarté du matin le plus pur. C'est cette dichotomie entre l'île rêvée (terre chérie d'un présent dont il serait enfin possible de jouir) et l'île réelle (terre honnie d'un présent qui disparait sans cesse sous le poids du passé) qui permet de lire Vasco et Touriste de bananes comme l'illustration du concept nietzschéen d'amor fati appliqué à une robinsonnade ontologique, c'est-à-dire à un récit qui établit la nécessaire et désirée transformation spirituelle du protagoniste comme moteur du séjour insulaire (Lojacono, 2014).

\section{Généalogie du désir insulaire}

L'interprétation nietzschéenne de Vasco comme de Touristes de bananes n'est pas le fruit du hasard, bien au contraire. Disons tout d'abord que les similitudes soulignées précédemment entre les trajectoires de Marc Chadourne et de Georges Simenon ne seraient pas tout à fait complètes si on ne faisait mention de leur commun intérêt pour la philosophie de Nietzsche. Dans un article publié en 1949 on ne s'étonnera pas de voir Chadourne insister sur l'aspect nietzschéen des œuvres de Gide. D'autre part, le Journal d'un journaliste de Robert de Saint Jean (1974), lui aussi reporter à ParisSoir dans les années trente, nous apprend de Chadourne qu'il « célèbre Havelock Ellis, Freud, son dieu étant Schopenhauer » (entrée du 23 novembre 1928) et il semble bien que le pessimisme de Schopenhauer eut une grande influence sur la vie de Vasco à 
ces débuts. Avant son départ, à Paris, il décrit ainsi sa vie faite de routines auxquelles il ne trouve aucun sens : « Ma vie fut à doses égales combinée d'horreur et d'ennui » (VS : 47), ce qui n'est pas sans rappeler la fameuse phrase de Schopenhauer : « La vie donc oscille, comme un pendule, de droite à gauche, de la souffrance à l'ennui » (Schopenhauer, 1912:326). Mais Vasco « tout congestionné qu'il était du Nietzsche de ses dix-huit ans » (VS : 30) choisit, contre Schopenhauer, de vivre dangereusement. Vasco est « une âme effrénée, sans Dieu, en quête de lumière », un homme qui « doit se surmonter pour apprendre le surhumain, c'est-à-dire toutes les latitudes de la vérité » (VS : préface VI). Une citation de Zarathoustra est en exergue de la troisième partie de l'œuvre, quant au surhumain et au dépassement de soi, ils nourrissent toutes les réflexions de Vasco aux derniers jours de sa solitude marquisienne.

Quand Simenon décrit ses jeunes années ne dirait-on pas là-aussi le portrait de Vasco : «J'ai lu toutes les œuvres de Nietzsche, en les annotant, à l'âge de dix-neuf ans et je les ai relu depuis lors » (Assouline, 2011 : 350). Anne Richter, dans Simenon sous le masque, consacre un chapitre à la tentation nietzschéenne de Simenon. On y apprend que « les paroles explosives du philosophe tombèrent sur ce jeune rebelle comme une lave : elles le marquèrent de façon indélébile » (2007: 39). La fougue et l'insolence de Zarathoustra, le romancier les retrouvait en lui-même, de même que le désir d'aller jusqu'au bout et on les retrouve aussi dans ses personnages, convaincus eux aussi d'aller jusqu'au bout d'eux-mêmes et « mus tôt ou tard par un même instinct auquel ils finissent inévitablement par obéir : celui de la route » (Richter, 2007 : 41). C'est ainsi que les personnages simenoniens ne se sentent vivre que lorsqu'ils partent. La fuite, thème de prédilection de Simenon, conjugué au retour à la nature des Adams de Chicago et des Ėves de Manchester, illustre selon Plamondon une tentation proprement nietzschéenne (Plamondon, 2009 : 52). Ni Vasco, ni Oscar ne réussiront cependant à surmonter leurs démons. L'échec du séjour polynésien tient à une erreur d'évaluation: Vasco et Oscar ont mal évalué leurs forces, mal évalué les motifs de leur fuite. Or, selon Nietzsche, c'est bien là tâche de philosophe, celle qui consiste «à résoudre le problème de l'évaluation, à déterminer la hiérarchie des valeurs » (Nietzsche, 1983 : 72). Une fois remonté à la racine rêveuse de leurs images poétiques, Nouhiva pour Vasco, la candide vision d'un toit rouge noyé dans la verdure pour Oscar, nous allons à présent en proposer la généalogie. Derrière le rêve de l'île, ce que Nietzsche appellerait « la métaphysique de l'île» avec toutes les connotations péjoratives qu'il attribue à la métaphysique, nous allons tenter de débusquer, comme le faisait le philosophe « les motivations idéalistes, caractéristiques de la décadence : la crainte du devenir, l'angoisse devant les instincts, la nostalgie d'un état de quiétude qui dispenserait l'homme de l'effort de la création » (Granier, 1982:40). La méthode généalogique entend ici faire l'historique des évaluations qui ont mené les deux protagonistes à l'échec, afin de mettre en lumière la source de leur erreur. Pour cela nous poserons une question simple : quelle est la valeur des valeurs attribuées par les protagonistes à la vie simple au fond des lagons couleur de béryl ? La fuite des protagonistes vers les rivages de la nouvelle Cythère pour y redécouvrir une vie plus 
authentique peut se lire comme le combat qu'ils livrent au spleen, comme l'ultime tentative pour échapper au pessimisme qui les détermine. Mais le combat est truqué, le ver est dans le fruit. L'idéal beau et propre qu'a toujours poursuivi Oscar, l'amour de l'autre pays de Vasco, ne sont que chimères et arrières-mondes.

Pour les deux protagonistes l'attrait de Tahiti répond à une motivation douloureuse qu'ils revêtent eux-mêmes des traits hospitaliers d'une terre lointaine (Lacassin, 1973 : 171). L'origine de leur fuite prend donc racine dans le terreau, fertile en ressentiments, d'une vie ressentie comme absurde au sein d'une famille, puis d'un monde, qui leur est étranger. La vraie nature du malaise est à chercher à l'intérieur des personnages, dans les replis secrets de leurs idéaux façonnés d'après la logique du ressentiment et donc inaptes à ouvrir la voie à une vie réellement nouvelle. Si Horanyi remarque bien que Touriste de bananes débute par l'évocation du malaise d'Oscar qui ne fera que s'amplifier pour devenir le leitmotiv du roman, selon elle, ce malaise n'aurait rien à voir avec le passé d'Oscar car il serait dû entièrement au contexte exotique (2013: 75). Or Oscar avait passé sa vie à braver le ridicule, à chercher des traces d'admiration dans le regard des autres. Sa timidité, sa lâcheté, il espérait les laisser derrière lui, mais le mépris de lui-même le poursuit à Tahiti. Rien n'a changé. À Paris, le besoin d'espace qui envahissait Vasco lui venait comme une suffocation et, à des milliers de kilomètres de distance, seul aux Marquises il constate que "rien n'a changé. Le poids n'est pas tombé » (VS : 241). Le contexte exotique ne fait que mettre à jour l'origine douloureuse de leur motivation. Oscar et Vasco, figures de l'exotisme nostalgique, sont des chrysalides du destin et «l'enfermement dans le cocon exotique précipite leur éclosion » (Moura, $1998: 349$ ).

L'exotisme n'est donc pas une cause mais un catalyseur. Dans sa hutte près de la cascade, Oscar se rend compte qu'il ne fait que s'agiter sans but. Alors ? Comment est-ce les Marquises ? demande le narrateur à Vasco. Alors rien. Le Vasco marquisien, comme le Vasco parisien est accablé par la petitesse de sa vie. À La Rochelle, Paris ou Papeete, l'homme qui ne sait pas se justifier lui-même souffre devant le problème du sens de la vie. Même si la vie est rudimentaire, proche de l'ascétisme des hommesnatures, cela ne change rien à la donne. Vasco, en proie à un pourquoi dont le tourment domine sa vie, et Oscar qui cherche depuis toujours à démontrer qui il est, ressentent « la profonde dépression, la pesante lassitude, la noire tristesse de l'homme psychologiquement atteint » (Nietzsche, 1983 : 197). Ce sentiment, assure Nietzsche, a des causes multiples comme par exemple le spleen européen ou le pessimisme du $\mathrm{XIX}^{\mathrm{e}}$ siècle. Vasco se place lui-même sous le signe de Mallarmé. Oscar, lui, est pareil à ceux qui partent un matin le cerveau plein de flamme / Le cour gros de rancune et de désirs amers mais cela ne fait pas de lui un voyageur. Vasco et lui font partie de ceux qui se croient voyageurs quand, rappelle Baudelaire, ils ne sont joyeux que de fuir une patrie infâme ou l'horreur de leurs berceaux. Un très intéressant article intitulé Le Pâle Vasco (Cellier, 1958) corrige de nombreuses erreurs de la critique à propos de ce poème et fait expressément le lien entre les deux poètes. Or les vrais voyageurs sont ceux-là seuls qui partent pour partir. Enchainés dans le carcan de leur 
famille, sans pouvoir extérioriser leur vraie nature, se développe en eux un profond état morbide qui correspond exactement à ce que Nietzsche dénomme la mauvaise conscience. Ils fuient leur famille en pensant ingénument éloigner ainsi le poids de l'hérédité. Ils ne veulent pas être de la race de ceux qui pèsent le pour ou le contre de toutes choses afin d'en bien mesurer les bénéfices possibles: "une telle race composée d'hommes du ressentiment finira nécessairement par être plus prudente que n'importe quelle race aristocratique, aussi honorera-t-elle la prudence en une toute autre mesure" (Nietzsche, 1983: 48). Et c'est justement les conseils de prudence de son père que Vasco a en horreur. Il se veut danseur léger aux flancs de la montagne, non «cul-de-plomb » au fond de rassurantes vallées comme son père (VS : 135, 268). Cette mauvaise conscience est celle-là même de l'homme du ressentiment, de celui dont la motivation est douloureuse et non pleine d'un gai savoir. C'est ainsi que bientôt « une relation de mal-être, un désir de fuite marquent leur rapport imaginaire à un lieu qui n'est pas senti comme le lieu réel» (Moura, 1998 : 267). Étrangers à leur famille, à leur pays, ils vont combattre ce malaise, non par un affrontement, dont ils n'auraient d'ailleurs pas la force, mais par la fuite. Leur fuite obéit à une émotion réactive et se range pour cela dans les actions de second ordre selon Nietzsche. Seul l'homme actif peut s'opposer à celui du ressentiment, car il est fort, spontané, agressif quand Oscar et Vasco sont, à leurs propres yeux, faibles, retenus, timides. Ils ne prennent aucune initiative, n'agissent pas en hommes libres mais réagissent en esclaves de leurs propres démons. Et partir n'a jamais signifié affronter ses démons. C'est là leur première erreur d'évaluation. Une seconde erreur sera de choisir leur destination à la clarté des lampes, en faisant de l'éloignement l'arc boutant de leur bonheur futur. En effet « la qualité première du lointain, l'étrangeté [...] permet de faire converger rêve et réalité. Là-bas il y a une amplification du possible du destin humain, un renouvellement peut-être merveilleux à connaître » (Moura, 1998 : 262). Leur destination sera donc lointaine. La chaleur émolliente des tropiques est, selon Nietzsche, le décor idéal du bonheur

tel que se l'imaginent les impuissants, les opprimés, accablés sous le poids de leurs sentiments hostiles et venimeux, chez qui le bonheur apparait surtout sous forme de stupéfiant, d'assoupissement, de repos, de paix, de "sabbat », de relâchement pour l'esprit et le corps, bref sous sa forme passive (Nietzsche, $1983: 47$ ).

Mais, ombre au tableau, de tant de splendeurs océanes suinte pourtant ce monstre délicat, l'ennui. Riccardo Pineri dans son essai L'île matière de Polynésie cite Touriste de bananes quand il parle de l'ennui qui s'exhale du séjour dans les îles (Pineri, 1992 : 92). On notera par ailleurs que le chapitre de son essai consacré au passage à la modernité s'intitule Le crépuscule des idoles, ce qui place ce texte dans la lignée du volume que Dorian Astor a publié sur Nietzsche en 2014. Le malaise qui avait présidé au départ des protagonistes, après une brève accalmie, réapparait à des milliers de kilomètres comme un gouffre qui les aspire. 
Il était là, debout au bord de l'océan, face au soleil qui déclinait et qui, dans un moment, toucherait la ligne d'horizon, il était là, dressé toute sa taille, bien d'aplomb sur ses jambes robustes et pourtant il avait une sensation angoissante d'inconsistance, ou peut-être de vide (TB : 337).

[...] cette douceur de l'île si envoutante, si lourde à porter cependant, où l'on sentait toujours, malgré tout, comme un vide, une absence, un trou dans le bonheur, que rien ne parvenait à combler (VS : 156).

En plein bonheur, Vasco note qu'il y avait dans l'air, parfois, une sorte de fatigue « une indécollable langueur, une torpeur amnésique » qui le gagnait : « euthanasie, petite mort douce et parfumée. Ah! Dieu, non, ce n'est pas un pays pour progresser !» (VS : 110). L'île est un espace ambivalent. Cythère, oui, mais Cythère morne ainsi que le fait remarquer Pierre Jourde (1997) en soulignant le côté morbide de ces îles qui n'ont de paradisiaque que le décor. La mort et le pourrissement des choses comme des mœurs est d'ailleurs un thème récurrent dans la littérature des îles à l'époque : «Tahiti, c'est un suicide, un suicide parfumé. Le plus voluptueux suicide du monde ... » (Margueron, 1989 : 207). La mélancolie, la déchéance, la maladie, le suicide, la mort, le cannibalisme sont autant d'impressions qui ont frappé des auteurs comme Stevenson, London, Adams, Gauguin, Loti, Gerbault et Dorsenne à propos de la Polynésie. Aux Marquises, deux jours avant de revenir sur sa promesse et d'abandonner lâchement le Frère à sa léproserie, Vasco note dans son journal : « C'est l'air d'ici. Tout y fermente, Tout y pourrit sous ces arbres. Il faut que j'aille pourrir aussi » (VS : 255). Une solution serait de combattre «ce malaise dominant par des moyens qui ramènent le sentiment de la vie à son expérience la plus rudimentaire » (Nietzsche, 1983 : 199). C'est ce que vont tenter Oscar et Vasco, en voulant vivre en harmonie avec la nature, eux qui n'ont jamais accepté de se voir comme des touristes de bananes et ont toujours refusé de devenir des clochards des tropiques. Le résultat de cette vie réduite à ses besoins minimaux nous dit Nietzsche, est antalgique, hypnotique : on aboutit à un anéantissement du moi, à une sanctification. Oscar sera le plus proche de cet anéantissement, lui qui voulait tant se fondre dans le glorieux paysage tropical. Vasco quant à lui tentera la voie de la sanctification. Mais là encore il y a eu erreur d'estimation car

une vie ascétique est une flagrante contradiction : un ressentiment sans exemple domine, celui d'un instinct qui n'est pas satisfait, d'un désir de puissance qui voudrait se rendre maitre, non de quelque chose dans la vie mais de la vie même, dans ses conditions les plus profondes les plus fortes, les plus fondamentales (Nietzsche, $1983: 177$ ).

Et toujours, le ressentiment en embuscade, la fatigue qui épuise, le passé qui étouffe.

\section{Zarathoustra aux îles}

L'aphorisme 151 du Gai savoir (Nietzsche, 2001 : 162) explique l'origine de la pensée métaphysique et dévoile en même temps la clé de la supercherie : les religions ont habitué les hommes à l'existence de mondes suprasensibles, et à la mort de Dieu, 
il n'est resté de cet immense espoir qu'amertume (d'où le ressentiment) et détresse (d'où la mauvaise conscience). Désormais cet autre monde, cet au-delà, vidé de ses dieux mais non de ses idoles, sera le domaine de la métaphysique. Pour les métaphysiciens, comme pour les prêtres, il y a deux vies : une vie ici-bas, vulgaire et absurde, et une autre, bien meilleure, dont l'accès sera permis sous certaines conditions. La pensée métaphysique contre laquelle s'est élevé Nietzsche est celle qui définit la vie en termes de dualismes: «La croyance fondamentale des métaphysiciens c'est l'idée de l'opposition des valeurs » (Nietzsche, 1953 : 13). Par conséquent est métaphysicien celui qui à chaque valeur terrestre fait correspondre une valeur supérieure du monde suprasensible : l'esprit et la matière, le beau et le laid, l'immuable et le changement et, bien évidemment, le bien et le mal. La croyance en un monde suprasensible, en un au-delà toujours meilleur que l'ici-bas, c'est ce que Nietzsche appelle l'idéalisme. Celui qui condamne la vie ici et maintenant, la vie telle qu'elle se présente à lui dans toute sa réalité au nom d'un idéal, comme le primitivisme par exemple, est appelé nihiliste. C'est pour cela qu'Oscar et Vasco sont des nihilistes au sens nietzschéen du mot, car, incapables d'apprécier la vie qui leur a été donnée, ils persistent à vouloir nier leur présent, à le contrecarrer, à le fuir, au lieu d'apprendre à l'apprivoiser, à défaut de pouvoir l'aimer. C'est au nom de l'idéal primitiviste qu'ils quittent la France, c'est au nom de ce même idéal qu'ils iront toujours plus loin, de Papeete vers les districts, vers les îles basses, vers les Marquises.

Nous avons déjà vu que l'île fonctionne comme un sur-lieu (Racault, 1989), nous pouvons à présent la définir comme un lieu métaphysique dont la particularité est de réunir, pour les protagonistes, toutes les "vraies » valeurs. Vasco et Oscar sont des êtres divisés entre devoir filial (pôle senti comme négatif) et aspirations personnelles (pôle senti comme positif). En chacun se joue « le duel hamlétique entre l'homme qu'il aurait voulu être et l'homme qu'il était» (VS : 267). À Fautaua, chez Plessis, Vasco « croyait voir s'accomplir son rêve : renaître, ressusciter, au bout du monde, nu sur une plage nu» (VS : 140). Quant à Oscar, ce qu'il voulait c'est «fuir les hommes et vivre d'une vie simple, labourer peut-être, apprendre, comme les indigènes, à capturer les poissons au harpon ...» (TB : 299). Devenir fort comme ceux de sa famille pour Oscar, devenir fort comme Plessis pour Vasco, pour tous deux devenir fort signifie ne plus subir le destin mais lui tenir tête, transmuer la faiblesse en force, la timidité en courage, la honte en affirmation de soi. C'est cela qu'ils sont venus chercher aux îles. « Mais il n'y a plus d'espoir. Je suis celui que je suis : comment saurais-je me débarrasser de moi-même ? Et pourtant — je suis las de moi-même !» (Nietzsche, 1983: 184). Devenir un autre, infiniment libre, infiniment fort, et pourquoi pas alors le plus grand de tous, celui qui règne sur les arrières-mondes? Aux Marquises, « il n'y avait que les crêtes et la mer tout autour de moi, la mer qui miroite aux quatre points cardinaux, avec ses taches d'îles perdues et sa buée ... La fin du monde. Ce jour-là, j'étais dieu ...» murmure Vasco (VS : 214). Oscar fait un bilan : «c'est pour cela qu'il était venu, pour forcer les poissons à la nage, pour redevenir, dans la nature, un animal harmonieux, pour mener la vie d'une sorte de dieu païen » (TB : 344). Ils n'ont pas brisé les idoles, causes de leur 
mauvaise conscience, ils se sont contentés de les remplacer. Se croire dieu c'est encore être dans la métaphysique, dans le culte des idoles, même si celles-ci ont apparemment changé de visage. L'exotisme ressortit donc à un nihilisme incomplet. L'enchantement n'aura duré que ce que dure le parfum du frangipanier un soir de tamuré car :

ni l'éternel soleil des îles, ni les lumières de Paris, ne suffisent à dissiper les ombres de la personnalité, ni à changer le sens de la vie. Sous les paysages qu'ils éclairent, l'existence s'écoule comme dans une serre, les rapports humains sont truqués ou effacés. Ce n'est pas la vraie vie, celle de tous les jours où il faut donner pour recevoir, se battre pour gagner, comprendre pour être aimé. D'où une déception, une mutilation, une démission qui rendent la fuite dérisoire ou inutile (Lacassin, 1973 : 170).

Or c'est cela même qu'ils cherchaient : changer le sens de leur vie. Mais la vie est partout la même pour ceux qui ne peuvent se changer eux-mêmes. À Paris, à La Rochelle, ils avaient la nostalgie d'un arrière-monde plus en conformité avec leur idéaux (la nostalgie correspond donc en premier à un manque) ; à Tahiti, ils se rendent compte que, pire encore que le manque, est « le manque du manque, c'est-à-dire l'état ou fait défaut tout objet désirable » (Stanek, $2010: 223$ ). L'île rejointe n'est plus objet de désir et c'est pourquoi les âmes des protagonistes oscillent douloureusement entre la souffrance (le manque) et l'ennui (la satisfaction de celui-ci). On comprend qu'ils aillent, comme Gauguin, porter leurs espoirs toujours plus loin de Papeete, dans les districts, jusqu'aux Tuamotu, jusqu'aux Marquises, afin de substituer à la douce torpeur de l'ennui les tiraillements acides d'un manque nouveau. Vasco essaie les danses et l'alcool mais son tourment profond avait déjà fait en lui tâche d'huile. Avant c'était le monde « vrai » qui manquait; à Tahiti c'est le « à quoi bon? » qui taraude. «Où vais-je ? Où tout cela me mènera-t-il ? À quoi bon cette inutile recherche du plaisir? Je fais fausse route » reconnait Vasco (VS : 156). Un soir, au retour de la pêche dans un village loin de Papeete, dans un moment de béatitude exaltante, Vasco est repris par ses démons : « On dirait [...] qu'il manque quelque chose. Mais quoi ? [...] Le goût du malheur » (VS : 156). Nostalgiques d'un passé tahitien qui n'est plus et n'a peut-être jamais existé, dans l'attente exténuante de l'avènement toujours remis d'un temps glorieux, à Moorea ou aux Marquises, Vasco et Oscar sont « des êtres qui, souffrant sans vouloir s'avouer ce qu'ils sont [...], s'étourdissent, se fuient euxmêmes et n'ont qu'une crainte : avoir conscience de ce qu'ils sont en réalité...» (Nietzsche, $1983: 225$ ). Les aspects quotidiens de leur vie ont changé certes, la palme a remplacé le chêne, mais ce n'est toujours pas la Vie. Et non seulement ce n'est pas la Vie mais les idéaux beaux et propres qu'ils avaient apportés avec eux dans leurs bagages ont été soit trahis, soit salis, et ce, avant même de débarquer : Tahiti est le pays du rhume, tout y est pourri, vous n'y serez qu'un raté parmi d'autres à vivre de bananes... leur avait-on dit et répété encore sur le bateau. Il leur aurait fallu pouvoir oublier car « nul bonheur, nulle sérénité, nulle espérance, nulle fierté, nulle jouissance de l'instant présent ne pourraient exister sans faculté d'oubli » (Nietzsche, 1983 : 76). Mais Oscar ne peut oublier, ni surmonter les humiliations du passé, Vasco ne peut oublier, ni admettre, qu'il ressemble à son père. Faute d'oubli, le pessimisme et le 
ressentiment refont toujours surface interdisant toute jouissance du moment présent, tout amor fati, et ce, sur une terre de plaisirs. Car à Tahiti il n'est que de jouir... «mais savoir jouir, tout est là... » reconnaît Vasco (VS : 110). Toujours en proie à leur mauvaise conscience, ils ne peuvent se contenter de la seule jouissance tout en étant inaptes au bonheur: "Que devenait alors le morsus conscientiae ? L'antithèse du gaudium » rappelle Nietzsche citant Spinoza (Nietzsche, 1983 : 117). Vasco et Oscar ont des parcours et des comportements très semblables : ils aspirent à se dégager du ressentiment dans lequel ils sont englués, l'un se raidit aux conseils de prudence de son père, l'autre contre sa faiblesse physique. Cependant, au paroxysme de leur mal-être, ils vont vivre pendant quelques instants la vie dont ils rêvaient, par procuration. Au procès du commandant Lagre, Oscar se dédouble et voit la meilleure part de lui-même affronter sans peur et avec une force tranquille les membres corrompus du tribunal. Enfin il se sent admiré de tous. Aux Tuamotu, puis aux Marquises, la raison de Vasco vacille et lui aussi se dédouble : est-ce lui qui agit ainsi ou est-ce Plessis ? Ayant pris la décision de remplacer le Frère à la léproserie, un autre personnage va accompagner ses derniers jours aux Marquises avant sa fuite, Zarathoustra. Se faire lépreux entre les lépreux signifie avoir trouvé la paix : «C'est la paix, Vasco, la vraie paix. Tu sais qui tu es maintenant. Un homme qui a été plus loin que les autres [...] Tu es sauvé ; tu t'es oublié » (VS : 259). Mais si la vie que lui proposait sa famille était trop étroite pour lui, trop mesquine, le surhumain que lui a enseigné Zarathoustra est trop grand, trop généreux. Il s'enfuira comme un lâche, laissant tout en plan.

\section{Conclusion}

Vasco sous le signe de Mallarmé et Touriste de bananes sous celui de Baudelaire, les deux romans se rejoignent au ciel plombé d'un spleen insidieux, qui, comme une pluie fine mais implacable, vous glace jusqu'aux os. Ni Vasco ni Oscar n'ont pu surmonter leurs démons (faiblesse, lâcheté, honte de soi) mais désormais ils sont allés trop loin, dans tous les sens du terme, pour se contenter d'une vie confortable et conformiste, à l'ombre castratrice de leur famille. Ils rejettent la vie prudente de l'homme modéré sans avoir eu la force de se surmonter eux-mêmes. La cause ? Partout ils ne voient qu'idéaux. Au nom d'une Vie qu'ils parent de tous les attraits, ils refusent la vie, celle de tous les jours, de tous leurs jours. La seule dont ils disposent. Toujours oscillants entre la douleur de leur filiation (le refus du passé) et leurs velléités de changement (les promesses du futur), ils sont condamnés à la détresse du présent. Alors? demande Nicou à Oscar, tournant le couteau dans la plaie. Alors ? demande le narrateur à son cousin, pourquoi es-tu revenu si à Nouhiva c'est si beau que tu dis ? Alors, répond l'internationale des ratés du Café du commerce, non seulement l'exotisme n'existe pas mais c'est un antidote frauduleux à la petite vie, un poison mortel au goût de frangipanier. À quoi bon vivre ? Vivre de bananes au fond d'une case perdue ou d'ortolans au cœur d'une cité tentaculaire sont les deux faces de la même monnaie. C'est ce que vont apprendre les protagonistes : vivre 
comme les hommes-natures et même dans le plus beau des paysages, ne résout pas le sens de la vie. En ce début du $\mathrm{XX}^{\mathrm{e}}$ siècle, Vasco et Oscar posent la question consubstantielle à l'homme moderne, la question du devenir : qu'allons-nous devenir, nous qui voulons nous maintenir, que ce soit de bananes ou des mets les plus fins? Dans ces deux romans, Chadourne et Simenon illustrent les débuts de la modernité qui

\footnotetext{
n'est pas une époque historique, c'est une manière de penser et de sentir que Nietzsche a décelé partout où il a perçu, à la source d'une détresse, une vaste entreprise de réduction de la multiplicité du monde de l'esprit, et une inflation de l'intériorité spirituelle au détriment de l'extériorité de ce monde (Astor, $2014:$ 201).
}

L'exotisme est donc une idole, un arrière-monde qui noie ceux qui ont eu l'inadvertance de s'y plonger. L'île paradisiaque est métaphysique car l'image du monde suprasensible et antinomique qu'elle véhicule empêche l'homme de rejoindre son être véritable, c'est-à-dire de vivre en paix avec ses propres contradictions. Ébahi par les couleurs de Tahiti, aveuglé par tant de lumière, l'homme moderne en oubli sa propre bigarrure. Choisir une île exotique pour tenter d'y vivre dans un monde dual, c'est-à-dire dans un monde en noir et blanc, choisir la profusion des formes tropicales pour nier la multiplicité des désirs et des possibilités vitales, c'est peut-être là tout le drame des touristes de bananes.

\section{RÉFÉRENCES BIBLIOGRAPHIQUES}

Alavoine, B., (1998) Georges Simenon. Amiens, Encrage Édition.

Assouline, P., (2011) Autodictionnaire Simenon. Paris, Librairie Générale Française. Astor, D., (2014) Nietzsche : La détresse du présent. Paris, Gallimard.

Bachimon, P., (2004) «L'image de Tahiti véhiculée par les artistes anglophones et francophones à l'époque coloniale », in The Journal of Pacific Studies. Vol. 27, no 1 , pp. 23-37.

Bertrand, A., (1994) Georges Simenon : de Maigret aux romans de la destinée. Liège, Éditions du CÉFAL.

Boucharenc, M., (2004) L'écrivain-reporter au cœur des années trente. Villeneuve d'Ascq, Presses Universitaires du Septentrion.

Cellier, L., (1958) «Le pâle Vasco », in Revue d'histoire littéraire de la France. № 4, Octobre-décembre, pp. 510-522.

Chadourne, M., (1928) « Chroniques », in La Revue européenne. Août 1928, pp. 858862.

Chadourne, M., (1949) «André Gide et l'humanisme », in The French Review. Vol. 22, n³, janvier, pp. 207-219.

Chadourne, M., (1994) Vasco. Paris, La Table Ronde.

Durand, P., (2000) «Formes et formalités. Remarques sur la poétique du nom chez Mallarmé », in Formules. Vol. 4, pp. 230-238. 
Granier, J., (1982) Nietzsche. Paris, PUF.

Horányi, L., (2013) «Les romans exotiques de Georges Simenon et le genre policier », in Lublin Studies in Modern Languages and Literatures. Vol. 37, pp. 69-81.

Jourde, P., (1997) « Cythères mornes », in Reig, D. (éd.), L'Île des merveilles. Mirage, miroir, mythe. Paris, L'Harmattan, pp. 193-208.

Lacassin, F., (1973) « Simenon et la fugue initiatique », in Lacassin F. \& G. Sigaux (éd.), Simenon. Paris, Plon, pp. 157-183.

Laissus, Y., (1978) « Catalogue des manuscrits de Philibert Commerson (1727-1773) conservés à la Bibliothèque centrale du Muséum d'Histoire naturelle (Paris) », in Revue d'histoire des sciences. Vol. 31, pp. 131-162.

Lecarme, J., (1989) « Les Romans coloniaux de Georges Simenon », in Textyles. Vol. 6, pp. 179-189.

Lojacono, F., (2007) « Rien de nouveau sous le soleil », in Francofonía. Vol. 16, pp. 51-65.

Lojacono, F., (2014) Roman de l'île et robinsonnade ontologique. Paris, Pétra.

Mallarmé, S., (1966) Poésies. Paris, Gallimard.

Margueron, D., (1989) Tahiti dans toute sa littérature. Paris, L'Harmattan.

Moura, J. -M., (1998) La Littérature des lointains. Histoire de l'exotisme européen au $X X^{e}$ siècle. Paris, Honoré Champion.

Nietzsche, F., (1953) Par-delà le bien et le mal : prélude d'une philosophie de l'avenir. Paris, Mercure de France, $10^{\text {ème }}$ édition.

Nietzsche, F., (1983) La Généalogie de la morale. Paris, Gallimard.

Nietzsche, F., (2001) Le Gai Savoir. Paris, Gallimard.

Pineri, R., (1992) L'île matière de Polynésie. Paris, Balland.

Piron, M., (1983) L'univers de Simenon. Guide des romans et nouvelles (1931-1972) de Georges Simenon. Paris, Presses de la Cité.

Pittman, L., (2010) « Marc Chadourne : Écrivain-voyageur ; 23 mai 1895 - 30 janvier 1975 », in Cahiers Robert Margerit. Vol XIV, pp. 146-163.

Plamandon, J. -F., (2009) « Exotisme et Touriste de bananes », in Rivista dell'Istituto di Storia dell'Europa Mediterranea. Vol. 2, pp. 47-58.

Racault, J.-M., (1989) « De l'île réelle à l'île mythique », in Moureau, F. (éd.), L'Île, territoire mythique. Paris, Aux Amateurs de Livres, pp. 79-99.

Richter, A., (2007) Simenon sous le masque. Bruxelles, Racine.

Saint-Jean, R., (1974) Journal d'un journaliste. Paris, Grasset \& Fasquelle.

Schopenhauer, A., (1912) Le Monde comme volonté et comme représentation. Burdeau, A. (trad.). Paris, Félix Alcan, $6^{\mathrm{ème}}$ édition.

Simenon, G., (2003a) « Touristes de bananes ou les dimanches de Tahiti », in Tout Simenon. Vol. 21, Paris, Omnibus, pp. 273-381.

Simenon, G., (2003b) La Mauvaise étoile. Paris, Gallimard.

Stanek, V., (2010) La Métaphysique de Schopenhauer. Paris, Vrin. 